\title{
Wie weiter mit Deutschland in der EU? Thesen zu den Gestaltungsaufgaben des Bundestages
}

\author{
von Werner J. Patzelt
}

Die „Euro-Rettungspolitik“ verdeutlicht, dass nicht nur die EU, sondern auch Deutschland an einem Scheideweg stehen und der Bundestag derzeit die Weichen für seine künftige Rolle in der Europapolitik stellt. Das Parlament als Vertretungsorgan des Volkes hat diese Grundsatzdebatte zu führen und die notwendigen Konsequenzen zu ziehen. Der Beitrag zeigt, welche Gesichtspunkte dabei zu erwägen sind und welche vorab $z u$ bedenkenden Konsequenzen sich mit den Handlungsalternativen verbinden. Deutschland hat einen ,europäischen Beruf", der auf absehbare Zeit durch einen nationaler parlamentarischer Kontrolle unterstellten Intergouvernementalismus sachdienlicher erfüllt wird als durch den Ausbau europäischer Supranationalität. Auf allen Ebenen muss die demokratische Kontrolle europäischer Politik verbessert werden. Die daraus erwachsenden Maximen für den Bundestag lauten: Verhinderung jeder Vergemeinschaftung europäischer Schulden, Sicherung dauerhafter Budgethoheit des Parlaments, Verbesserung des europapolitischen Selbstbestimmungsrechts des deutschen Volkes durch Einführung fakultativer Referenden für die Ratifikations- und Begleitgesetze zukünftiger Vertragsänderungen, Hinwirken auf gleiches Stimmengewicht bei Wahlen zum Europäischen Parlament und dauerhafte Verhinderung eines präsidentiellen Regierungssystems auf EU-Ebene.

The on-going efforts to "rescue" the Euro demonstrate that Germany stands at a crossroads. The Bundestag is currently defining its future role in European politics. As the people's representative body, it has to face this fundamental debate and needs to draw the necessary consequences. This contribution shows which aspects need to be considered and which consequences can be said to arise from different choices. Germany has a "European profession" that can, for the foreseeable future, be better fulfilled by an intergovernmental EU, kept in check by national parliaments than by an extension of European supranationality. Democratic oversight needs to be improved at all levels of government. The guidelines for the Bundestag should therefore be: refrain from any mutualisation of European debt, secure lasting sovereignty over national expenditures, improve the selfdetermination of the German people in European politics by introducing facultative referenda on all future Treaty changes, work towards equal weighting for all votes at European Parliament elections, and avert the introduction of a presidential system of government at the EU level. 


\section{Der Teil und das Ganze}

Es bringt wenig, ein impressionistisches Gemälde aus allzu großer Nähe zu betrachten - man erkennt nur Farbkleckse, nicht aber jene größeren Zusammenhänge, die ihren eigentlichen Sinn ausmachen. Auch setzt ein Maler seine Pinselstriche besser mit Blick auf jenes Ganze, dem sie Ordnung, Aussage und Leben verleihen sollen. Kaum anders ist es bei der Analyse politischer Institutionen und bei der Sorge um jene Regeln, die sie ausmachen. Erst recht bedarf es des Blicks auf das größere Ganze, wenn einer schon bestehenden Einrichtung in einem werdenden oder sich wandelnden Institutionengefüge ihr Platz zugewiesen oder gesichert werden soll. So verhält es sich zurzeit mit dem Parlament einer Bundesrepublik Deutschland, die wieder einmal ihre Rolle im sich stark verändernden Europa suchen muss.

Bei den - zusammengenommen durchaus spektakulären - Entscheidungen des Bundesverfassungsgerichts der letzten Jahre, Monate und Wochen zur Rolle des Bundestages im europäischen Regierungssystem und zum rechten innerstaatlichen Umgang zwischen Bundestag und Bundesregierung geht es nämlich um weit mehr, als der Blick nur auf den jeweiligen Fall erkennen lässt. ${ }^{1}$ Gewiss richtet sich die Aufmerksamkeit rechtlich vornehmlich auf die europäische Integrationsverantwortung des Bundestages, auf dessen ausschlaggebendes Wort bei der - nicht nur, aber gerade auch haushalterischen - Staatsleitung, auf die

1 Mit Stand vom 3. Juli 2012 handelt es sich Wesentlichen um folgende Entscheidungen: Urteil vom 19. Juni 2012 im Organstreit „ESM/Euro-Plus-Pakt“, zurückgehend auf eine Klage der Fraktion Bündnis 90/Die Grünen gegen eine Verletzung der Unterrichtungsrechte des Bundestages durch die Bundesregierung im Zusammenhang mit dem Europäischen Stabilitätsmechanismus (ESM) und dem EuroPlus-Pakt; Urteil vom 28. Februar 2012 im Organstreit „Beteiligungsrechte des Bundestages/ESFS“ gegen die Verlagerung wichtiger haushaltspolitischer Beteiligungsrechte von Abgeordneten des Bundestages im Rahmen des europäischen Stabilisierungsmechanismus auf ein kleines Sondergremium des Haushaltsausschusses, zurückgehend auf Klagen von zwei Bundestagsabgeordneten; Urteil vom 30. Juni 2009 zum Vertrag von Lissabon und seinen Begleitgesetzen, zurückgehend auf Klagen des Bundestagsabgeordneten Peter Gauweiler (CSU), der Bundestagsfraktion der Linken sowie weiterer Abgeordneter; Urteil vom 12. Februar 1993 zum Vertrag von Maastricht, dessen Ratifikationsgesetz und die mit ihm einhergehende Verfassungsänderung, zurückgehend auf Klagen mehrerer Politiker und Staatsrechtler. Im gleichen Zusammenhang stehen die zurückgewiesenen Verfassungsbeschwerden bzw. Anträge auf einstweilige Anordnungen in den Urteilen vom 7. September 2011 gegen Maßnahmen zur Griechenlandhilfe und zum Euro-Rettungsschirm, vom 22. Juni 2011 gegen die Übernahme von Gewährleistungen im Rahmen des europäischen Stabilisierungsmechanismus sowie vom 8. Mai 2010 zur Verhinderung der Gewährleistungsübernahme für Kredite an Griechenland. Wegweisend wird ferner das Urteil zu den unlängst vorgelegten Eilanträgen gegen die Ausgestaltung des Euro-Rettungsschirms sowie den europäischen Fiskalpakt sein. Im Lauf der Jahre betonten diese Entscheidungen immer mehr Grenzen der Übertragung deutscher Hoheitsrechte auf die EU sowie die Verantwortung des Bundestages bei der Kontrolle des europapolitischen Gebarens der Bundesregierung. 
Informationspflichten der Bundesregierung gegenüber dem deutschen Parlament und auf die institutionellen Artikulations- wie Mitwirkungsrechte seiner Mitglieder. Doch im nächstgrößeren Zusammenhang geht es machtpolitisch um den Einfluss der nationalen Parlamente im supranationalen Regierungsprozess der EU sowie ihrer Sonderbünde, v.a. der Eurozone und des Schengen-Gebiets. Letztlich und strategisch aber steht die Frage nach der Rolle im Mittelpunkt, die die Nationalstaaten - und allen voran Deutschland - in einer sich mit ungewissem Ergebnis wandelnden EU spielen sollen bzw. können.

Die überwölbende und alles entscheidende Frage ist die letztgenannte. Unentrinnbar konkret wird sie für die deutsche Staatspraxis in den erstgenannten Herausforderungen, die mit den Mitteln des Staatsrechts zu bestehen sind. Als Puffer dient ein Bundestag, der zwischen einem überzeugungsgetragenen „Weiter so!“ einer großen Mehrheit seiner Abgeordneten und dem immer deutlicher spürbaren Misstrauen vieler Deutscher gegenüber der europapolitischen Weitsicht ihrer regierenden Klasse laviert. Der Bundestag laviert, weil zwar weichenstellende Entscheidungen über den zu gehenden Weg anstehen, doch nicht nur die innere Bereitschaft, sondern bislang auch der äußere Zwang fehlt, sie als wirklich grundsätzliche Entscheidungen zu treffen. Also wird ,auf Sicht gefahren“, d.h. sich in gewohnter Richtung vorsichtig nach vorn bewegt, ohne an unübersichtlichen Punkten einen Orientierungshalt zum gründlichen Kartenstudium einzulegen. Aufgrund der europäischen Schulden- und Währungsprobleme beschleunigt sich die Fahrt sogar noch, wenn auch begleitet von Warnsignalen des Bundesverfassungsgerichts als derzeit wichtigstem Hüter deutscher Eigenstaatlichkeit. Das alles dramatisch zu nennen, ist keinesfalls übertrieben. Es geht nämlich um nicht weniger als die weitere Ausgestaltung von Deutschlands ,europäischem Beruf“. Was ist damit gemeint?

\section{Deutschlands „europäischer Beruf“}

Im mittleren 19. Jahrhundert wurde zur Identitätsfrage Preußens, was in die Formel von „Preußens deutschem Beruf“ gefasst war und auf eine anstehende Entscheidung verwies: Sollte Preußen - zum Königreich erhoben und inzwischen, neben Österreich, des Deutschen Bundes mächtigster Staat - fortan seinen Weg im losen Gefüge dieses Bundes weitergehen, oder sollte der Hohenzollernstaat das kleinteilige Deutschland über diesen Bund hinausführen, also die deutsche Integration zu seinem Hauptanliegen machen? Das freilich hätte zwei Konsequenzen. Erstens wäre der Vielvölkerstaat Österreich, da nicht in einen deutschen Nationalstaat passend, aus dem wieder zum Reich werdenden Bund zu 
drängen, was ohne kriegerische Auflösung des preußisch-österreichischen Dualismus zugunsten einer preußischen Führungsrolle kaum gelänge. Zweitens würde im Staatenverbund zwischen dem großen Preußen und dem viel kleineren, zudem sehr zerstückten ,,mittleren Deutschland“ der preußische Staat machtpolitisch deckungsgleich mit dem neuen Deutschland werden. Auf diese Weise würde Preußen gleichsam ,in Deutschland aufgehen“. Das ablehnend, sperrte sich nicht nur der preußische König noch am Vorabend der Reichsgründung gegen die Forderung, Preußen habe - und sei es um den Preis der Identitätsaufgabe einen ,deutschen Beruf“ zu erfüllen.

Die Sache mit Deutschlands ,europäischem Beruf“ verhält sich einesteils sehr anders. Weder dominiert Deutschland die EU so wie einst Preußen den (Nord-) Deutschen Bund, noch gibt es irgendeinen Dualismus zwischen zwei „,europäischen Vormächten“ auszutragen. Auch hat sich Deutschland hinsichtlich Europas noch viel weniger ausgesucht, dass ihm nun ein solcher „Integrationsberuf“ zukommt, als das einst so absichtsvoll expandierende Preußen. Der ,europäische Beruf" ist unserem Land ganz einfach zugewachsen: aufgrund seiner Mittellage in Europa und seiner unglücklichen Größe (,,zu groß für ein Gleichgewicht, zu klein für die Hegemonie“ ${ }^{\text {2 }}$ ) - was ein vereintes Deutschland ohne supranationale Einbettung zum Risiko für Europas geopolitische Balance und dadurch für sich selbst macht -, zugewachsen auch dank der supranationalen Tradition des Alten Reiches (dessen Funktion als „Friedenswahranstalt“ die EU unter neuen Umständen so erfolgreich übernommen hat), und zugewachsen nicht zuletzt aufgrund der großen Tradition (west-)deutscher Europapolitik, die sich einst - auch mangels sinnvoller Alternativen - vorbehaltlos auf die Einigung Europas einließ und mit der Wiedervereinigung des Landes unter Zustimmung seiner Nachbarn eine gewaltige Friedensdividende dieser Politik einstreichen konnte.

Andernteils ist unverkennbar, dass sich nun die so lange offengehaltene Frage ganz praktisch stellt, ob Deutschland für die absehbare Zukunft lieber eine eigenständige Nation in einem „Europa der Vaterländer“ sein will, oder ob es sich eher eine Zukunft als Gliedstaat einer Europäischen wünscht, deren Eigenstaatlichkeit bis zur Aufsicht über die nationalen Haushalte von Mitgliedsländern reicht. Wie unerhört solcher Zugriff auf den harten Kern staatlicher Selbstbestimmung wäre, zeigt ein Vergleich der Eurozone mit Deutschland. Trotz langer staatlicher Einheit samt gemeinsamer Währung wird in unserem Land eine wirk-

2 S. Dehio, L.: Gleichgewicht und Hegemonie. Betrachtungen über ein Grundproblem der neueren Staatengeschichte, Krefeld, 1948 (Neuausgabe: Darmstadt, 1996). 
same Kontrolle der Landeshaushalte durch die Bundesregierung als inakzeptabel empfunden. Sie wird, sogar angesichts verfassungsrechtlicher Schuldenbremsen, auch gar nicht ernsthaft diskutiert - obwohl manche Bundesländer strukturell kaum lebensfähig sind und die übrigen gewaltige Schulden tragen. Auch gibt es innerdeutsch keinerlei Bereitschaft zur wechselseitigen Haftung für die Schulden der öffentlichen Hand. Doch eine auf beiderlei Weise vergemeinschaftete Haushaltspolitik behandelt man sehr wohl als zentrale Option weiterer europäischer Einigungspolitik, ja empfiehlt sie sogar als Voraussetzung für den - vielfach sehr erwünschten - Übergang der Eurozone zu jener Transferunion, die die Bundesrepublik Deutschland mit ihrem horizontalen und vertikalen Finanzausgleich längst wurde. Ist es aber wirklich das Gleiche, Bremen aus Bayern und Griechenland aus Deutschland querzusubventionieren?

Nicht minder deutlich ist angesichts Deutschlands „europäischem Beruf“, dass von unserem Land gerade in der aktuellen Eurokrise politische Führung verlangt wird. Das aber geschieht meist in der Erwartung, dass Deutschland die EU gerade nicht dorthin zu führen versucht, wohin Deutschland will, sondern nur in eine Richtung, die den Geführten gefällt. An deren Ende soll offensichtlich keine Agenda 2010-Politik oder verfassungsrechtliche Schuldenbremse nach deutschem Vorbild stehen, sondern eine Dauerrolle Deutschlands als europäisches Garantie- und Geberland, darin ähnlich dem Status Baden-Württembergs in Deutschland. Die unvermeidlichen Folgelasten für Deutschland, so die Hoffnung, sind durch konsequentes Beschweigen aus der gegenwärtigen Diskussion herauszuhalten. Zu ihnen zählt auf mittlere Frist das Absinken deutscher Bonität an den Kreditmärkten auf ein europäisches Durchschnittsniveau, was Deutschlands Haushaltsspielräume und somit Gebermöglichkeiten deutlich beschränken und alsbald jene Konflikte umso heftiger anfachen wird, die man jetzt noch in aufgezwungener Spendabilität zu umgehen sucht. Langfristig aber wird in der Eurozone und der EU jene wechselseitige Missstimmung um sich greifen, die im Nachgang unfair empfundener Vertragsbeziehungen bei gleichzeitig - trotz Art. 50 EUV - fehlenden (da kaum praktikablen) Kündigungsmöglichkeiten noch immer ausgelöst wurden. Am Ende dürften die Neubelebung von Nationalismen und die Erosion des Erreichten stehen.

Solche auf den ersten Blick ganz paradoxe Verhaltenserwartungen sind freilich gut nachvollziehbar. Man muss nur auf die Nachkriegsrolle (West-)Deutschlands blicken. Dieses Land hatte sich von Anfang an der europäischen Einigung verschrieben - nicht nur, doch freilich auch, weil diesem besiegten und verstümmel- 
ten Teilstaat von der Siegermächte Gnaden keine andere Politik bessere Perspektiven bot. Aber (West-)Deutschland hatte sich diese Politik auch sehr redlich zueigen gemacht, einesteils der Friedens- und der Wohlstandssicherung willen, und andernteils, um ein halbwegs berechenbares außenpolitisches Umfeld zu konstruieren. Nicht zuletzt aus Staatsräson trieb (West-)Deutschland die europäische Integration entschlossen voran, notfalls auch unter Einsatz großer eigener Finanzmittel, und behandelte jedes Zurückbleiben der EGKS, EWG, EG und EU hinter einer ,ever more perfect Union“ als Folge von Unzulänglichkeiten gerade auch der eigenen Politik. Warum also sollten Deutschlands europäische Partnerländer gerade jetzt unter, ,deutscher Führung“ einen anderen Kurs wünschen, da doch entlang des eingeschlagenen Wegs immer mehr deutsches Geld, deutsche Kredite und deutsche Bürgschaften zu immer günstigeren Bedingungen locken?

Nur überholen die an Deutschland gerichteten finanziellen Hoffnungen erstmals die realistischerweise erwartbare deutsche Leistungskraft. Auch dürfte die Verwirklichung solcher Hoffnungen bald die Hinnahmebereitschaft der deutschen Bevölkerung überfordern. Außerdem wird jede vom Bundestag zu beschließende Transferleistung an gleich welchen „Eurorettungsfonds“ - so die derzeit immerhin in Aussicht gestellte Praxis - das Schwinden solcher Hinnahmebereitschaft beschleunigen. ${ }^{3}$ Sowohl aus wirtschaftlichen als auch aus innenpolitischen Gründen drängt sich jedenfalls die Frage auf, wie lange unser Land seinen Kurs des europapolitischen ,Weiter so!“ beibehalten kann - oder gar eines integrationspolitischen ,plus ultra!“, wie es einst der Wahlspruch Kaiser Karls V. forderte. Das wiederum führt zur Frage, wann Deutschland endlich klären will, ob es ein aufgrund eigener Gestaltungsmöglichkeiten innerhalb der EU agierender Nationalstaat sein will oder ob es im europäischen Staatenverbund aufgehen soll, nämlich als ein in wesentlichen Dingen nicht länger zum eigenen Entscheiden fähiges ,europäisches Bundesland“.

Auf dem letztgenannten Weg erfüllte Deutschland seinen „europäischen Beruf“ am Besten durch entschlossenes Hinwirken auf die Schaffung der „Vereinigten Staaten von Europa“. Das war auch lange Zeit eine populäre deutsche Vision, ihrerseits nicht selten verknüpft mit dem Gebot politischer Korrektheit, an Deutschland eher zu leiden und es loswerden zu wollen als dieses Land zu mö-

3 Bereits jetzt wird öffentlich die Empfindung artikuliert, deutsche Führung gemäß den Wünschen der Geführten liefe auf eine Art nachgeholter Reparationspolitik hinaus; s. hierzu die Debatte um Sarrazin, T.: Europa braucht den Euro nicht. Wie uns politisches Wunschdenken in die Krise geführt hat, München, 2012. 
gen. ${ }^{4}$ Wer dieses Ziel hingegen für unrealistisch oder gar nicht wünschenswert hält, sollte freilich auch nicht jene Schritte verlangen, die nur auf dieses Ziel hin einen sinnvollen Weg ergeben. Tatsächlich brächte die Aufgabe eigenständiger deutscher Handlungsmöglichkeiten ohne Schaffung eines demokratisch legitimierten europäischen Regierungssystems unser Land um seine effektive Staatlichkeit und das deutsche Volk um seine Demokratie. Wer das nicht will, muss deshalb das Fortbestehen handlungsfähiger und für sich selbst verantwortlicher Nationalstaaten mit zwar einer wirkungsvollen, doch durch das Subsidiaritätsprinzip beschränkten EU zu verbinden versuchen. Deutschlands „europäischer Beruf" bestünde dann nicht darin, unser Land in Europa aufgehen zu lassen, sondern in der zielstrebigen Schaffung und Aufrechterhaltung eines europäischen Regierungssystems, das die Nationalstaaten zwar überwölbt, doch nicht entmündigt.

Jede der zwei Weisen, Deutschlands „europäischen Beruf“ auszuüben, kann man mit guten Gründen wollen. Doch man kann nicht beiden Wegen folgen. Bei der deshalb erforderlichen Entscheidung, welcher Kurs einzuschlagen sei, muss sicher eine große Rolle spielen, dass Deutschland sowohl aufgrund seiner Mittellage als auch wegen seiner prekären Größe aufs Neue in die gefährliche politische Lage des Kaiserreichs geriete, wenn seine inzwischen gelungene Einbettung in die EU als Gemeinschaft befreundeter Staaten Schaden nähme. Deshalb ist jede Politik unverantwortbar riskant, die ein Auseinanderbrechen der EU wahrscheinlich macht. Nicht minder ist aber zu berücksichtigen, dass Deutschlands Nachbarn, durch die Geschichte gründlich belehrt, um dieses Dilemma unseres Landes wissen und es auch zum eigenen Vorteil nutzen. Außerdem zeigt sich, dass nicht nur ein ,zu wenig“, sondern auch ein ,zu viel“ an Integrationspolitik zum Risiko des Auseinanderbrechens führt - zumindest dann, wenn aus guten Gründen das Vertrauen schwindet, die sich aufeinander in der Eurozone oder im Schengen-Gebiet einlassenden Staaten würden ihre politischen Bringschulden wirklich begleichen. Und das heißt: Welche Summen Deutschland in die EuroRettungspolitik investieren sollte, lässt sich nur dann mit Vernunftgründen entscheiden, wenn vorab festgelegt wurde, ob Deutschland ein eigenständig handlungsfähiger Staat bleiben oder eine supranational regierte Wirtschaftsregion innerhalb der Eurozone werden soll.

4 Aufs Deutlichste sichtbar machte diese Haltung der Bundespräsident Gustav Heinemann, der - gefragt, ob er Deutschland liebe - unter allgemeiner Zustimmung die Antwort verweigerte und ins ganz Persönliche auswich: Er liebe seine Frau. 
Solange Europa noch wirkungsvoll zwischen westlichen Demokratien und realsozialistischem Block gespalten war, wirkte Deutschlands geopolitisches Dilemma wie ein dankenswerterweise vergangenes und mit Blick auf Gegenwart wie Zukunft vollständig theoretisches Problem. Doch eine Generation nach dem Ende der europäischen und deutschen Teilung wurde es wieder zum Thema höchst praktischer Politik. Deutschland reagierte auf die Neuentstehung jenes Problems mit weitblickender Fortsetzung des Bewährten, nämlich mit vertiefender und erweiternder europäischer Integration. Die Kurzformel für diese auf „Euro und EU-27“ hinführende Politik war seit je, dass die deutsche und die europäische Einigung zwei Seiten derselben Medaille seien. Nur wie weit die Einigung Europas wirklich gehen sollte, blieb offen - und konnte bis zur Gegenwart wohl auch offen bleiben.

Zwar gab es seit Beginn der europäischen Integrationspolitik Diskussionen um die - ehedem selten so genannte - „Finalität“ des entstehenden Gebildes. Dass mehr als ein institutionell gesichert zusammenarbeitendes ,Europa der Vaterländer" nicht werden solle, war etwa für die meisten Franzosen eine Selbstverständlichkeit. Nicht so aber für viele Deutsche, die im Grunde nichts sehnlicher wünschten, als ihre schimpflich gewordene Nation ,in Europa“ abstreifen zu können, um - wie gleichsam vor dem Sündenfall der preußischen Reichseinigung - im Kleinen wieder Hanseaten oder Hessen, im Großen aber nichts anderes als Europäer zu sein. Dieser Zielkonflikt konnte unentschieden bleiben, solange noch nicht über die (mit der NATO-Integration erreichte) Vergemeinschaftung des Militärs hinaus ebenfalls an eine Vergemeinschaftung zunächst der Währung, sodann der Staatsschulden, anschließend der Haushaltspolitik und am Ende auch der Wirtschafts- und Sozialpolitik als der „harten Kerne“ speziell demokratischer Staatlichkeit zu denken war. Die Einführung des Euro, absichtsvoll eingesetzter Hebel für die weitere politische Integration Europas, hat diesbezüglich deren Geschäftsgrundlage ganz wesentlich verändert. Schon viel weniger gilt dies aber für das jeweils nationale Geschäftsgebaren. In der Konsequenz steht die aktuelle „Krise der Eurozone“. Sie löst unabweisbaren Handlungs- und Klärungsdruck aus, seit - eigentlich von vornherein absehbar - jener Schutzdamm gebrochen ist, der einst in der Zusicherung bestand, kein Staat werde für die finanziellen Risiken eines anderen Staates haften müssen und insofern die Verfügung über den eigenen Staatshaushalt einbüßen. Inzwischen ist derlei Haftung eingeführt, obendrein ohne zeitliche Begrenzung. Und sobald diese Haftungspflichten - wohl demnächst - sich in entsprechenden Geldabflüssen aus Deutschland niederschlagen, alsbald die verfassungsrechtliche Schuldenbremse 
wirkungslos machen und dann auch zum fremdverursachten Rückbau unseres Sozialstaats führen, wird auch den europapolitisch besonders gutmütigen Deutschen klar werden: Jetzt geht es an den Kern der demokratisch gestaltbaren Eigenstaatlichkeit.

In dieser Lage verkettet sich wieder einmal Deutschlands geopolitisches Dilemma mit den Varianten seines ,europäischen Berufs“. Wird nämlich die so gewagt unternommene Währungsunion zurückgenommen auf jene Länder, welche die für sie erforderliche Haushalts-, Wirtschafts- und Sozialpolitik zu führen bereit sind, dann dürfte das nicht nur als Korrektur, sondern auch als Rückschritt empfunden werden - zumal von jenen Staaten, denen die Zinsvorteile des Euro und die Haushaltsbürgschaften anderer Euro-Länder nutzen. Deren Enttäuschung über die Erosion der Eurozone wird gewiss Erbitterung gegen jene Staaten nähren, die - wie Deutschland oder die Niederlande - sich anschließend von einer drückenden Last befreit fühlen. Gemeinsam mit der ebenfalls anstehenden und kaum geringer enttäuschungsträchtigen Verkleinerung des derzeit sich nicht wirkungsvoll gegen illegale Einwanderung schützenden Schengen-Gebiets dürfte das eine Stimmung des Scheiterns herbeiführen, die ihrerseits weitere Zerfallsrisiken der EU zeitigen mag. Wahr ist freilich auch, dass jetzt schon die Eurozone die EU spaltet, weil unter dem Dach gemeinsamer Institutionen ganz offen Sonderbundspolitik betrieben wird. Und zu bedenken ist, dass der - längst begonnene - Zerfall der EU in ein „Europa mit variabler Geometrie“ uns schon mittelfristig wieder die geopolitische Lage des Kaiserreichs bescheren kann.

Kommt es aber umgekehrt zum Wandel der Eurozone zur Transferunion mit dank europäischer Kontrolle nationaler Budgets - fast vollendeter Supranationalität, dann droht unser Land wieder auf Zeiten unabtragbarer Staatsverschuldung oder stabilitätsgefährdender Inflation zuzugehen. Zwar ist unwahrscheinlich, dass gerade im Konfliktfall ein EU-Gremium einem nationalen Parlament dessen Budgetrecht wirksam entziehen kann. Bloße Rechtstitel nutzen ja nichts, wie in Euroland das Schicksal des einst vereinbarten wechselseitigen Haftungsausschlusses zeigt. Steht so viel Geld und damit die nationale Selbstbestimmung auf dem Spiel, wird deshalb alles auf reale Machtproben hinauslaufen. Konkret ist zu erwarten, dass der erste Machtkonflikt dieser Art zur Eskalation gebracht wird, sobald die Schulden der europäischen Staaten - gleich in welchem Rechtsgewand - effektiv vergemeinschaftet sind. Dann wird man einerseits kein Mitgliedsland mehr wirkungsvoll dazu anhalten können, auf weitere Schuldenaufnahme zu verzichten. Dem wird ein vollständiger Vertrauensverlust unter den Euro-Ländern folgen. Andererseits wird nach Vergemeinschaftung der europäi- 
schen Schulden Deutschland zusammen mit manch anderen Euro-Ländern, die zuvor eine halbwegs solide Haushaltspolitik führten, niederdrückende Lasten zu tragen haben, was sich innenpolitisch nicht lange durchhalten lässt. Also wird es dann entweder zu einem weiteren Sonderbund jener Staaten kommen, die sich nicht länger für die Fehler anderer in Haftung nehmen lassen wollen, oder es wird ein deutscher Vertragsbruch das europäische Einigungswerk erst recht erschüttern. Zunächst wird also die Legitimität der deutschen Demokratie, sodann die Stellung Deutschlands in Europa zerrüttet sein. In beiden Fällen kehren viele Gespenster der Vergangenheit zurück.

Die schlechteste Amtsführung wäre es, in dieser Lage die Dinge einfach treiben zu lassen. Teil des Treibenlassens ist es aber, deutsche Europapolitik vor allem pragmatisch-situativ zu gestalten und im Konfliktfall maßgeblich an den Wünschen der Nachbarstaaten auszurichten. Besser wäre eine erneute, grundlegende Reflexion darauf, was Deutschland in Europa wollen soll und was die deutsche Wählerschaft wollen wird. Seinen „europäischen Beruf“ kann Deutschland auf verschiedene Weise ausüben - nur nicht auf alle unterschiedlichen Arten zugleich. Also muss sich unser Land wieder einmal entscheiden. Das aber bedeutet institutionell: Der Deutsche Bundestag, im Wesentlichen die ständige Versammlung unserer nationalen politischen Klasse und einziges Staatsorgan mit direkter demokratischer Legitimation, muss solche Entscheidung öffentlich erwägen, treffen und den Deutschen vermitteln. Soweit es nicht um - parlamentarisch zu kontrollierende - Prärogativaufgaben der Bundesregierung geht, hat allein der Bundestag das erste Wort zu sprechen. Und falls er gute Gründe findet, unser Land über die Grenzen seiner derzeitigen Verfassungsordnung und demokratischen Eigenstaatlichkeit hinauszuführen, muss er es möglich machen, dass das letzte und entscheidende Wort von den Bürgern selbst gesprochen wird, nämlich in einer Volksabstimmung. Noch fehlt es im Bundestag anscheinend am Willen, diese sehr weitreichenden Reflexions-, Entscheidungs- und Vermittlungsaufgaben so gründlich zu erfüllen, wie es unserer Lage angemessen wäre. Sehr ähnelt unser nationales Parlament - und nicht nur seine regierungstragende Mehrheit einem „willigen Helfer“ der vom europäischen Tages- und Marktgeschehen gehetzten Bundesregierung. Das aber läuft hinaus auf Pflichtvergessenheit jener Institution, der - in Ernst Friesenhahns trefflicher Formulierung - die Staatsleitung gemeinsam mit der Bundesregierung ,zur gesamten Hand“ anvertraut ist. 


\section{Der Bundestag zwischen vollendeter Supranationalität und kon- trolliertem Intergouvernementalismus}

\section{Die Grundentscheidung}

Willensbildung zum ,europäischen Beruf“ Deutschlands, gegründet auf Responsivität gegenüber dem Volk und mündend in - durchaus konkurrierende - Führungsanstrengungen: Das ist die konkret anstehende und bislang unzureichend erfüllte europapolitische Aufgabe des Bundestages. Niemand anderes als er hat zu klären, welche Stellung er selbst in Europa haben will, welche Stellung dann aber auch unser Land in Europa haben muss, und welche Machtkämpfe es in diesem Fall mit einer Bundesregierung auszufechten gilt, für die ja nicht nur die deutschen Parlamentarier, sondern auch die vielen Regierungen der EU maßgebliche Mitspieler mit unangenehmen Vetomöglichkeiten sind. Deshalb ist es nicht so, als würden vom Bundestag gezogene ,rote Linien“ die Verhandlungsposition der Bundesregierung immer nur schwächen. Ganz im Gegenteil lädt gerade das Fehlen klarer „roter Linien“ Deutschlands Partner zum durchaus vernünftigen Erkunden jener Grenzen ein, an welche die Bundesregierung gehen kann. Wird aber der Grenzverlauf zu einer Frage taktischen Geschicks, so ist es eher fahrlässig, wenn der Bundestag die Grenzziehung der Tagesform der Kanzlerin oder den Zufälligkeiten einer Verhandlungsnacht überlässt. Dem Volk gegenüber gehört sich solche Fahrlässigkeit umso weniger, als diesem bislang jedes Instrument fehlt, jene europapolitischen Entscheidungen von Regierung und Parlament zu korrigieren, deren Konsequenzen es mehrheitlich nicht tragen will. Seit also spätestens aufgrund der Einführung des Euro - die Europapolitik von einem Spezialfeld der Außenpolitik zur erweiterten Innenpolitik wurde, läuft es auf eine schwere Beeinträchtigung unserer Demokratie hinaus, wenn nicht einmal die Volksvertretung für die Klärung und Durchsetzung dessen sorgt, was unser Land in Europa wollen soll und unser Volk dann auch zu tragen hat.

Will er nicht pflichtvergessen die Dinge weiter treiben lassen, hat also der Bundestag zwischen zwei Weisen zu wählen, auf die sich Deutschlands „europäischer Beruf" ausüben lässt. Erstens kann der Bundestag die Bundesregierung darin unterstützen, dass sie mit möglichst vielen EU-Partnerländern die Weichen auf ein demokratisches und echt supranationales europäisches Regierungssystem stellt. Zweitens kann der Bundestag darauf hinwirken, dass Deutschland diesen Weg gerade nicht geht, sondern den zu Deutschlands Nutzen erreichten Zustand nachhaltig sichert, nämlich durch Rückbau des allzu Wagemutigen und durch Ausbau des noch Unzulänglichen. Zum Letzteren gehört die gemeinsame Si- 
cherheits- und Außenpolitik, zum ersteren die Verkleinerung der Eurozone und des Schengen-Gebiets. ${ }^{5}$ Die Grundentscheidung ist also die zwischen einer EU mit vollendeter Supranationalität und einer EU, die auf der Grundlage des bislang erreichten supranationalen Integrationsniveaus einen viel wirkungsvoller parlamentarisch-demokratisch kontrollierten Intergouvernementalismus betreibt. Hinsichtlich des Bundestages führt die Entwicklung im ersten Fall zu kaum etwas Anderem als dem Absinken unseres Parlaments auf die Machtmöglichkeiten eines „europäischen Landtags“. Im zweiten Fall hat der Bundestag die Chance, zum mitgestaltenden Vetospieler in einem „Europa der Vaterländer“ zu werden, das seinem gemeinsamen Regierungssystem gerade keine eigene Staatsqualität verleiht.

\section{Zwei Spielarten vollendeter Supranationalität}

Ein vollständig ausgebautes supranationales Regierungssystem der EU kann, wenn es nachhaltig funktionieren soll, entweder als ein parlamentarisches oder als ein präsidentielles Regierungssystem ausgestaltet werden. Der Entstehung aller Zwischenformen sollte sich der Bundestag wegen der mit ihnen einhergehenden Risiken legitimitätsgefährdender Systemblockaden in den Weg stellen.

Als parlamentarisches Regierungssystem sollte die EU sinnvollerweise über folgende Institutionen verfügen: Erstens braucht es ein Europäisches Parlament, das nicht nur jederzeit die EU-Kommission stürzen kann, sondern - vor allem den Kommissionspräsidenten allein nach parlamentarischem Mehrheitswillen wählen kann, ggf. auf Erstvorschlag des Rates der EU - doch unbedingt mit der Freiheit, nach Ablehnung dieses Vorschlages auch einen Kommissionspräsidenten nach eigenen Wünschen zu wählen. Zweitens wäre die Europäische Kommission - durchaus auch mit Europaparlamentariern besetzt - zu einer echten europäischen Regierung weiterzuentwickeln, deren Präsident - und gerade nicht der Präsident des Rates der EU - dann auch den Titel eines „Europäischen Präsidenten“ führen sollte. Drittens müsste die Zusammensetzung des Europäischen Parlaments so geändert werden, dass - anders als derzeit - jede Wählerstimme über das gleiche Ergebnisgewicht verfügt. Nur eine bevölkerungsproportionale Repräsentation machte nämlich jenen großen Machtzuwachs des Europäischen Parlaments demokratisch akzeptabel, der mit der Supranationalisierung des europäischen Regierungssystems einhergeht. Viertens braucht es die Beschränkung

5 Die tollkühne Erweiterung der EU um die Türkei scheint ohnehin nicht mehr tatkräftig vorangetrieben zu werden, nicht einmal von der Türkei selbst. 
des Rats der EU, derzeit als Versammlung von Staats- und Regierungschefs die tatsächliche Regierung der EU darstellend, auf das - ja durchaus beträchtliche Machtniveau des Deutschen Bundesrates als einer Föderalkammer im europäischen Regierungssystem.

Selbst unter - fünftens - strikter Einhaltung des Subsidiaritätsprinzips, überwacht von einem weiterhin starken Europäischen Gerichtshof, ist dann nach wenigen europäischen Legislaturperioden ein Absinken der nationalen Parlamente auf das Machtniveau deutscher Landtage zu erwarten. Zum einen verlieren die nationalen Parlamente ihrerseits jenen Einfluss auf das europäische Regieren, den die - von ihnen innerstaatlich kontrollierten - nationalen Regierungen genau dann aufgeben, wenn sie nur noch in der Föderalkammer eines europäischen Parlaments agieren können. Zum anderen würde, aufgrund des sich gewiss auch im europäischen parlamentarischen Regierungssystem rasch einspielenden Dualismus zwischen kommissionstragender Parlamentsmehrheit und Opposition, bald ein nicht nur nominell europäisches Parteiensystem entstehen. Aufgrund ihrer realen Machtrolle als europäischer Spitzenparlamentarier würden dessen Führer, anders als heute, auch in den nationalen Parteisystemen Führungsrollen erlangen können. Das aber würde die europäische Parteipolitik bald so sehr mit der jeweils nationalen Parteipolitik verschränken, dass nicht nur eine dichte demokratische Legitimation europäischer Parteipolitik entsteht, sondern diese auch auf den „europäischen Politikfeldern“ die nationale Parteipolitik so überlagert, wie das heute in Deutschland die bundespolitische Parteiebene mit der landespolitischen Parteiebene tut.

Die Folge wäre, dass eine für die interessierte Öffentlichkeit auch individuell erkennbare europäische politische Klasse entsteht. Das wiederum führte zum Aufkommen einer wirklich ,europäischen Öffentlichkeit“, nämlich einer solchen, die dauerhaft auf das fortan sowohl personalisierbare als auch dramatisierbare europäische Regierungssystem bezogen ist. Weil aber parlamentarische Macht sehr stark auf Öffentlichkeit beruht, stiege damit auch die Macht des Europäischen Parlaments im Vergleich zur Macht der nationalen Parlamente, stiege also die Macht der europäischen politischen Klasse im Vergleich zur Macht der jeweils nationalen politischen Klassen. Das alles stellte sich außerdem umso schneller ein, je mehr Politikfelder tatsächlich der Zuständigkeit des europäischen Parlaments und der von ihm getragenen Kommission unterstellt würden womöglich bis hin zur tatsächlichen Kontrolle der Haushalte von Mitgliedstaaten. Im Grunde wäre das jene enge politische Union, die mit der Einführung des Euro „,von der ökonomischen Basis her“ erzwungen werden sollte. Wer zur 
Überwindung der Euro-Krise ,mehr Europa“ fordert, sollte die jeweils empfohlenen nächsten Schritte ganz von diesem Zielzustand ableiten.

Schwer ist zu bestreiten, dass ein sich so stark wandelndes europäisches Regierungssystem die Rahmenbedingungen deutscher Staatlichkeit weit über die Intentionen und Bestimmungen des Grundgesetzes hinaus veränderte. Bereits sich auf diesen Weg zu einem supranationalen Regierungssystem zu machen, verlangt deshalb - sofern man es mit demokratischer Legitimation politischer Herrschaft ernst meint - über eine bloße verfassungsändernde Mehrheit im Bundestag hinaus ein Tätigwerden des pouvoir constituant. Das wäre zumindest eine Volksabstimmung, der entsprechende Veränderungen der europäischen Verträge unterworfen werden müssten. Womöglich wäre sogar eine verfassunggebende Nationalversammlung einzuberufen, um Deutschland mit einer Verfassung auszustatten, die wirklich zu dieser veränderten Rolle des deutschen Staates in Europa passt. Gesetze zu geben, die entweder weichenstellende europapolitische Volksabstimmungen ermöglichten oder zur Wahl einer verfassunggebenden Nationalversammlung führten, wären dann zwingend zu erfüllende weitere Aufgaben des Bundestages.

Einen anderen Weg zu institutionell schlüssiger Supranationalität des europäischen Handlungsverbundes weist das Ordnungsmodell des präsidentiellen Regierungssystems. Soll ein solches Regierungssystem nachhaltig gut funktionieren, ${ }^{6}$ wäre eine Orientierung am US-amerikanischen Regierungssystem anzuraten, weil dieses nicht nur das bislang bestandsfähigste, sondern obendrein das einzige bis heute freiheitssichernde präsidentielle Regierungssystem darstellt. Um der EU ein ähnliches Regierungssystem zu schaffen, reichten fünf Maßnahmen. Erstens müsste die Institution eines von den europäischen Völkern direkt gewählten Europäischen Präsidenten geschaffen werden. Zweitens wäre die Kommission der Absetzbarkeit durch das Europäische Parlament zu entziehen und als reiner Regierungsstab - außerdem ganz ohne einen hervorgehobenen Kommissionspräsidenten - unmittelbar dem Europäischen Präsidenten zu unterstellen. Drittens braucht es ein Europäisches Parlament mit bevölkerungsproportionaler Zusammensetzung, weil nur dieses ein demokratisch schlüssiges Gegengewicht zum Präsidenten bilden kann. Viertens müssten auch hier die nationalen Regierungen eine mit dem Europäischen Parlament bei der europäischen Rechtssetzung zusammenwirkende Föderalkammer bilden, ansonsten aber, vom Subsi-

6 Wie leicht ein präsidentielles Regierungssystem entgleisen kann, ist trefflich untersucht in Linz, J.: The Failure of Presidential Democracy, Baltimore, 1994. 
diaritätsprinzip wirkungsvoll gegen Übergriffe der europäischen Ebene geschützt, auf das Regieren ihrer Mitgliedsländer beschränkt werden. Fünftens hätte ein starker Europäischer Gerichtshof über die Einhaltung des Subsidiaritätsprinzips zu wachen.

Auch in einem so aufgebauten präsidentiellen Regierungssystem dürfte sich bald eine individuell erkennbare europäische politische Klasse sowie eine auf sie fokussierte Öffentlichkeit herausbilden. Gerade auf sie muss sowohl der Europäische Präsident als auch das Europäischen Parlament seine Legitimität gründen. Doch anders als in einem parlamentarischen Regierungssystem ist sehr fraglich, ob es auch zur Entstehung eines effektiven europäischen Parteiensystems kommen kann. Ein Europäischer Präsident wird an seiner Integrations- und Führungsaufgabe im nationendiversen Europa wohl scheitern, wenn er sich vor allem als Parteiführer in Szene setzt. Und ohne den - einem präsidentiellen Regierungssystem ganz fremden - Dualismus zwischen regierungstragender Mehrheit und Opposition geht auch vom Parlament kein sonderlicher Anreiz für die Entstehung eines europäischen Parteiensystems aus. Dann aber wird ebenfalls die Herausbildung eines politisch belastbaren Zusammengehörigkeitsgefühls und Elitenverbunds der europäischen Völker zweifelhaft. Letzteres aber bräuchte es, weil die einem Europäischen Präsidenten und Parlament zu übertragenden Kompetenzen doch gewiss die Außenpolitik und die Sicherheitspolitik umschlössen, desgleichen die Prärogative bei finanzpolitischen Gestaltungsaufgaben, sofern sie die Stabilität der - zumindest etlichen Mitgliedsländern gemeinsamen - Währung betreffen. Eben dadurch aber setzte die europäische Ebene auch schon die Rahmenbedingungen nationaler Wirtschafts- und Sozialpolitik. Auf allen diesen Politikfeldern sammelte sich wiederum so viel Sprengstoff, dass ihn wohl nur ein sowohl vertikal als auch horizontal gut integrierendes Parteiensystem entschärfen kann. Fehlt es, dann entstehen rasch Regierbarkeitsprobleme. Im Übrigen wird ein mit vielen nationalen Vetomöglichkeiten ausgestaltetes präsidentielles Regierungssystem in der EU wegen der hieraus resultierenden Politikblockaden nicht funktionieren. Reduziert man aber die nationalen Vetomöglichkeiten, dann beschränkt das auch die nationalen Parlamente auf ihre rein innenpolitische Macht. Also wird es in einem arbeitsfähigen präsidentiellen Regierungssystem der EU erst recht zu ,europäischen Landtagen" in den Mitgliedstaaten kommen.

Nun muss es keine bestürzenden Vision sein, dass die Französische Nationalversammlung und der Deutsche Bundestag sich einst, verglichen mit dem Europäischen Parlament, in der politischen Gewichtsklasse des Landtags von Nordrhein- 
Westfalen wiederfinden. In Deutschland nimmt man solche Machtabstufungen zwischen dem Deutschen Bundestag und dem Saarländischen Landtag ja auch als Normalität hin - und desgleichen, dass im Bundesrat die Landesregierungen alles, die Landtage aber so gut wie nichts zu bestimmen haben. Allerdings gab es schon in der - so gern ohne national identifizierendes Beiwort auskommenden „Bundesrepublik“ ein ganz selbstverständliches Zusammengehörigkeitsgefühl der Bevölkerung, nämlich als Deutsche durch Abkunft oder Einbürgerung, das bei politischen Fragen sehr klar die regionale Identifikation als Rheinländer oder Hanseat überwog. Doch es ist sehr zu bezweifeln, dass ein ähnlich starkes europäisches Zusammengehörigkeitsgefühl bald auch in den zur EU gehörenden Nationen entstehen wird. Eher auf das Gegenteil deuten die kulturellen Nebenschäden der Euro-Krise hin. Ohne ein sich zusammengehörig empfindendes europäisches Volk hat aber weder eine supranationale Volksvertretung noch ein Europäischer Präsident eine im Konfliktfall belastbare Machtbasis.

Also sollte man besser nicht erwarten, dass sich die EU mit einem präsidentiellen Regierungssystem wirklich gut regieren kann. Dann aber darf man den Weg zu diesem Regierungssystem gar nicht erst einschlagen. Mithin wäre zu fordern, dass der Bundestag jede Weichenstellung dorthin unterbindet und schon jetzt signalisiert, dass er keinem europäischen Vertrag zustimmen würde, der die Institution eines volksgewählten europäischen Präsidenten schüfe. ${ }^{7}$ Die Devise muss lauten: Wenn europäische Supranationalität, dann nur in der Form eines parlamentarischen Regierungssystems.

\section{Intergouvernementalismus mit verbesserten Kontrollmechanismen bei gleichbleibendem Integrationsniveau}

Doch wäre die Vollendung europäischer Supranationalität für die EU wirklich der richtige Weg? Offen bleibt immerhin, wie deren noch weiter supranationalisiertes Regierungssystem sich mit jenen Sonderbünden vertrüge, die inzwischen als Eurozone oder als Schengen-Gebiet entstanden sind und sich unweigerlich in einem „Europa der verschiedenen Geschwindigkeiten“ vermehren. Schon auf dem jetzigen Integrationsstand kommt es zu großen rechtlichen und praktischen

7 Das muss auch für den Fall gelten, dass man diese Institution „rein symbolisch“ meint, also auch einem volksgewählten Europäischen Präsidenten ebenso wenige Befugnisse einräumt wie dem jetzigen Präsidenten des Europäischen Rates. Dann würden nämlich auch die Präsidentschaftswahlkämpfe zur reinen Symbolik und müssten alsbald Zweifel am Sinn solcher Wahlen und am Demokratiegehalt des europäischen Regierungssystems zeitigen. 
Problemen, wenn das Handeln der jeweils unterschiedlichen Beschlussgremien miteinander koordiniert und unter das Dach des supranationalen Regierungssystems gebracht werden muss. Tatsächlich treibt jenes „Europa mit variabler Geometrie", das allein den - sehenden Auges hingenommenen - Widerspruch zwischen der erfolgten Erweiterung der EU und ihrer in Teilen wünschenswerten Vertiefung erträglich machen kann, ganz von selbst zum Intergouvernementalismus als Normalmuster europäischer Politik. Dieser wurde aus gutem Grund überall dort zur Praxis, wo - seit dem Maastrichter Vertrag von 1992 - die EU außerhalb der Gründungsverträge zu agieren begann. Trotz der Supranationalisierungsschritte der Verträge von Amsterdam (1997) und Lissabon (2007) vollzieht sich wirkungsvoller Intergouvernementalismus weiterhin unübersehbar in der Gemeinsamen Außen- und Sicherheitspolitik (GASP) sowie im Agieren der Euro-Gruppe. Zwar gab es bei der GASP markante Supranationalisierungsbemühungen, etwa die Einführung eines „Hohen Vertreters für Außen- und Sicherheitspolitik“, der zugleich als Vizepräsident der Kommission fungiert. Trotzdem fügt sich die GASP immer noch recht gut in die traditionelle Trennung von Innen- und Außenpolitik (mit reduzierter parlamentarischer Kontrollmöglichkeit im letztgenannten Bereich). Hingegen trifft das Agieren der Euro-Gruppe mit Wucht die Haushalts- und Innenpolitik, die Wirtschafts- und Sozialpolitik und somit alle Eigenstaatlichkeit in ihrem demokratisch gestaltbarem Kernbereich. In dieser Lage gibt es wirklich gute Gründe für das Verlangen nach mehr nationalstaatlicher parlamentarisch-demokratischer Kontrolle des Regierungshandelns in europäischen Gremien.

$\mathrm{Zu}$ diesen Gründen zählt nicht nur die Sorge um die Eigenstaatlichkeit der Euroländer. Vielmehr zeigte sich schon vor den Euro-Rettungsmaßahmen, dass „Exekutivföderalismus" auch im Rahmen der EU keine anderen Wirkungen hervorbringt als im Rahmen Deutschlands: Es geraten die Parlamente der Gliedstaaten in Gefahr, recht nachrangige Institutionen für jene sich immer weiter ausdehnenden Politikfelder zu werden, in denen Regierungsgremien europäischer Sonderbünde den Ton angeben. Solcher Machtabstieg ist zwar nicht so unvermeidlich wie im Rahmen einer vollendet supranationalen EU. Doch es gibt auch beim Intergouvernementalismus keine Umkehrmöglichkeit auf dem Weg nach unten, sobald es Verfassungspraxis wird, dass die nationalen Parlamente ebenso wenig europapolitisch mitbestimmen können wie die deutschen Landtage in der Bundespolitik. Schon in den Gründungsjahren der Bundesrepublik Deutschland war den Landtagen nur wenig Erfolg bei ihren halbherzigen Versuchen beschieden, das Bundesratsverhalten ihrer Landesregierungen parlamentarisch zu kontrollie- 
ren, und inzwischen gilt es als ganz selbstverständlich, dass landesparlamentarische Arbeitszeit nicht durch bundespolitische Debatten verschwendet werden sollte. Den von der Sache her gleichen Kampf um die parlamentarische Kontrolle des Verhaltens der Bundesregierung in EU-Gremien führt derzeit eine kleine, heterogene Minderheit von Bundestagsabgeordneten.

Doch anders als mancher ehedem aufbegehrende Landtag findet sie bislang Rückhalt beim Verfassungsgericht. $\mathrm{Zu}$ den Ursachen zählt einerseits, dass die reale Eigenstaatlichkeit Deutschlands doch etwas anderes ist als die schon nach dem Zweiten Weltkrieg nur noch legalfiktive Eigenstaatlichkeit der deutschen Länder. Mit solcher Eigenstaatlichkeit aber ist das Demokratieprinzip dahingehend verknüpft, dass auch nach außen gerichtetes Staatshandeln nicht ohne Kontrolle und Einfluss der Volksvertretung bleiben darf. Andererseits balanciert auf europäischer Ebene kein starkes Parlament und keine parlamentsgetragene Regierung die Macht der Staatenregierungen so aus, wie das auf Bundesebene der Handlungsverbund von Bundestag und Bundesregierung gegenüber dem Bundesrat sehr wohl tut. Deshalb litt in Deutschland die Demokratie keine Not, als es im immer unitarischer werdenden Bundesstaat zur Kompetenzausdünnung der Landtage bei großem föderalem Machtzugewinn der Landesregierungen kam. Auf europäischer Ebene aber gibt es - und zwar gerade in einem Sonderbund wie der Eurozone - kein verlässlich wirkungsvolles Gegengewicht zur Politik jenes Exekutivenverbundes, der sich im jeweiligen Gremium der Staats- und Regierungschefs verstetigt. Es fehlt sowohl am Widerlager einer Kommission, die Fleisch vom Fleisch eines die europäischen Bevölkerungen proportional vertretenden Europäischen Parlaments wäre, als auch - in Deutschland abgesehen vom Bundesverfassungsgericht - an wirkungsvollen innerstaatlichen Gegengewichten. Praktisch unterläuft die Euro-Rettungspolitik, obwohl von überragender europapolitischer Bedeutung, sowohl die bestehenden supranationalen Einrichtungen der EU als auch hierzulande - so zumindest das Bemühen der Bundesregierung - die normale Kontrollpraxis des Deutschen Bundestages. Im Grunde sitzt das Parlament in der Politikverflechtungsfalle und leidet dort an Verantwortungsdiffusion.

Beide Defizite lassen sich, langen Atem vorausgesetzt, aber durchaus korrigieren. Erstens wäre der demokratisch-parlamentarischen Kontrolle auf europäischer Ebene eines Tages sehr geholfen, wenn der Bundestag bald zu erkennen gäbe, er wolle weiteren europäischen Vertragsänderungen nur dann zustimmen, falls die Weichen hin zu einem bevölkerungsproportionalen Europäischen Parlament gestellt würden. Allein schon eine angemessen große deutsche Stimmen- 
zahl im Parlament stärkte das Gewicht der Bundesregierung bei europäischen Verhandlungen. Zweitens zwingt die nationalen Parlamente der EU nichts dazu, sich der Europapolitik ihrer Regierungen so unterzuordnen, als gehe es um „normale Außenpolitik“, bei der sich das Parlament mit Blick auf die gouvernementale Verhandlungsfähigkeit zurückzunehmen hat. Ganz im Gegenteil schuf sich der Deutsche Bundestag inzwischen Informations- und Kontrollmöglichkeiten, die ihn zum wirklich einflussreichen Mitspieler machen können. ${ }^{8}$ Doch im Zusammenwirken der - unserem Regierungssystem angemessenen - Fraktionsdisziplin mit einer grundsätzlich pro-europäischen Einstellung der meisten Abgeordneten entfalten die europapolitischen Führungsvorgaben der Bundesregierung enorme Sogwirkung, und zwar selbst dann, wenn ein großer Teil der Parlamentarier durchaus nicht mehr glaubt, eine bestimmte Regierungsposition sei ,alternativlos“.

Drittens sollte der Europapolitik des Bundestages nicht mehr jener demokratische Schild fehlen, den Volksabstimmungen schaffen können: Nicht die Bundesregierung, nicht der über die Bundesregierung weitergeleitete Druck anderer Staaten könnte den Bundestag zu einer anschließend wirklich bindenden europapolitischen Entscheidung zwingen, sobald das Volk die Möglichkeit besäße, binnen bestimmter Frist, gestützt auf eine Mindestanzahl an Unterschriften zugunsten einer Referendumsinitiative, in einem fakultativen Referendum über das entsprechende Ratifikations- oder Begleitgesetz des Bundestages zu befinden. Allein schon die Vorauswirkung eines - im Fall unpopulärer Politik auch nur drohenden - Referendums würde die Rolle des Bundestages gegenüber der Regierung und die Position der Bundesregierung gegenüber ihren EUPartnerländern stärken. Also sollte der Bundestag dem von ihm vertretenen Volk dieses plebiszitäre Instrument nun bald geben. Alle drei Maßnahmen gemeinsam ließen den immer wichtiger werdenden Intergouvernementalismus in der EU zu einem national und demokratisch kontrollierten Intergouvernementalismus werden, und zwar ohne jede Gefährdung für den Bestand des derzeit unvollendeten supranationalen Regierungssystems.

8 S. Demuth, Chr.: Der Bundestag als lernende Organisation. Eine evolutionstheoretische Analyse der Lern- und Anpassungsprozesse des Bundestages, insbesondere an die europäische Integration, BadenBaden, 2009. 


\section{Europapolitische Aufgaben des Bundestages}

Solange es aber weder eine wirklich demokratische europäische Supranationalität noch europapolitisch geeignete plebiszitäre Instrumente in allen Mitgliedstaaten gibt, entstehen sowohl auf der europäischen als auch auf der nationalen Ebene ernstzunehmende Demokratielücken europäischen Regierens. Sie werden zur eigentlichen „Legitimitätslücke“ des europäischen Einigungswerks, sobald die europäischen Völker - und zumal die finanziell so sehr geforderten Deutschen den Eindruck gewinnen, es werde in der EU bewusst über ihre Staaten, Volksvertretungen und nationalen Interessen hinwegregiert. Solche Empfindungen aber breiten sich derzeit aus - nicht nur in Deutschland, sondern auch in Staaten wie Griechenland. Angesichts seiner aktuellen und kommenden Herausforderungen ist der gegenwärtige Zustand des europäischen Regierungssystems also durchaus unbefriedigend. Aus der Warte des intergouvernemental in die Enge getriebenen Deutschland und der sehr eingeschränkten demokratischen Mitsteuerungsmöglichkeiten seiner Bevölkerung ist die Lage sogar dramatisch.

Einen gangbaren Ausweg scheint die weitere Supranationalisierung der EU aber nicht zu bieten - so reflexhaft auch gefordert wird, am Ende ebenfalls dieser Krise müsse wieder einmal „,mehr Europa“ stehen. Denkbar wäre zwar der Versuch eines Durchstartens zum parlamentarischen Regierungssystem auf europäischer Ebene. Doch es ist nicht zu erkennen, dass Staaten mit nach 1989 neu errungenen nationalen Selbstbestimmungsmöglichkeiten, wie die Tschechische Republik und Polen, oder immer schon unabhängige stolze Nationen, wie das Vereinigte Königreich und Frankreich, in einem solchen supranationalen Europa wirklich so aufgehen wollen, wie sich das immer noch viele Deutsche zu wünschen scheinen. Also dürfte der Weg zu noch mehr Supranationalität in der EU bis auf weiteres eine Sackgasse darstellen. In die aber sollte man sich nicht verirren, sondern es mit verstärktem Intergouvernementalismus im Rahmen des bestehenden Regierungssystems versuchen. Erreichbar wäre auf diesem Weg sogar eine Stabilisierung des Geschaffenen durch den Rückbau von Überzogenem sowie durch die Entschleunigung intergouvernemental aufgezwungener Innenpolitik durch verbesserte parlamentarische und plebiszitäre Regierungskontrolle. Der Weg zu künftig vollendeter Supranationalität, eines Tages vielleicht wieder wünschenswert, wäre damit nicht verstellt. Nur käme eine „vertiefte Integration“ dann nicht wie eine „oktroyierte Verfassung“ des 19. Jahrhunderts, sondern gründete auf redlichem und weit verbreitetem Verlangen nach einer ,more perfect Union“. 
Dieser Vorschlag scheint in Deutschland aber noch nicht mehrheitsfähig zu sein. Denn erstens prägt die deutsche politische Klasse bis heute der aufrichtige Wunsch, unser Land zum Teil eines supranationalen Europa zu machen. Eben deshalb billigte der Bundestag noch jeden weiteren Integrationsschritt, selbst wenn viele Abgeordnete über eine allzu freigiebige Europapolitik ihrer Regierung murrten - wissend um die zunehmende Spannung zwischen dem plus ultraKurs der Regierung und dem status quo-Verlangen der vom Staunen zur Skepsis übergehenden Bevölkerung.

Zweitens setzt sich hierzulande gerade in Krisenzeiten immer wieder das Argumentationsmuster durch, jetzt sei nicht die Stunde nationaler Eigensucht, sondern weiteren europäischen Zusammenwachsens. Einerseits ist das eine ganz traditionelle und bewährte deutsche Argumentationsfigur. Andererseits ist es aber so, dass seit der Osterweiterung der EU nicht nur aus der Mitte der Gesellschaft, sondern gerade auch aus ihrem rechtem Rand sich immer heftiger die Sorge artikuliert, es werde die Einschränkung nationaler Kontrolle von Zuwanderung, Grenzsicherung, Verwendung des Staatseinkommens und währungssichernder Haushaltspolitik die deutsche Staatlichkeit und demokratische Selbstbestimmung über die Grenzen des Zumutbaren hinaus untergraben. Seither wird solche Skepsis ob weiterer europäischer Integration in der Öffentlichkeit immer öfter als unbedingt zu bekämpfende ,rechte Position“ etikettiert und gilt immer weniger als eine Lagebeurteilung, die man mit Vernunftgründen erörtern sollte. Stattdessen prägen Erwägungen politischer Korrektheit oder zielführender innenpolitischer Taktik immer öfter jenes Nachdenken über deutsche Staatsräson, um das es hier doch geht. Und bereits das Denken entlang dem Begriff der Staatsräson wird immer wieder als ,,rechts“ und ,deshalb zu überwinden“ kritisiert.

Solches Zusammenwirken von pro-europäischer Grundhaltung und anti-rechter Ablehnung aller Integrationsskepsis schuf dann - drittens - jenen Einvernehmenszusammenhang, der am Beginn der ,Euro-Rettungspolitik“ stand. Denn obwohl nun für die jahrelange Defizitpolitik eines anderen Eurolandes vertragswidrig in Haftung genommen, sagten Regierung und Bundestag in großer Eile ihr ,Ja“ zu Bürgschaften und - aller Wahrscheinlichkeit nach - eines Tages fällig werdenden Verbindlichkeiten in einer Höhe, über die selbst nachzudenken deutsche Sozial- oder gar Verteidigungspolitiker bei ihren Budgetforderungen nicht wagen. Solch grundsätzlicher Hilfsbereitschaft, seither bei immer weiter steigenden Summen immer lauter eingefordert, wurde rasch und argumentativ schlüssig die Forderung zur Seite gestellt, man müsse das zu kurierende Übel nun endlich ,an der Wurzel packen“. 
So kam es - viertens - zur Forderung, in der Eurozone der innerstaatlichen Budgethoheit nationaler Parlamente supranationale Schranken zu setzen, im Gegenzug dann aber auch die Verantwortung für vergangenes - und erwartbar künftiges - budgetäres Fehlverhalten zu vergemeinschaften. Eine solche Forderung nach verbindlichen Spielregeln für alle ist allzu verständlich aus der Warte eines Landes, das mit einer „Politik der freien Hand“ sowie zwei Hegemonialversuchen sehr schlechte Erfahrungen gemacht hat, sich deshalb seit 1949 gern supranationalen Strukturen einfügt, dabei aber doch unverkennbar eigene Vorstellungen vom richtigen Haushalten und Wirtschaften über die eigenen Grenzen hinaus verbindlich zu machen hofft. Dieser Forderung wäre freilich gegenzurechnen, dass nicht alle europäischen Staaten Deutschlands Vorfreude auf Supranationalität teilen, zumal dann nicht, wenn sie - oft wesentlich weniger machtvoll als Deutschland - gar nicht damit rechnen können, in Europas supranationalem Regierungssystem wahrnehmbaren Einfluss ausüben zu können. Als Reaktion zeitigt solch hochgemuter Supranationalismus denn auch wenig anderes denn die klassische balance of power-Politik: Unter dem Beifall etlicher weiterer Staaten spielten bei Brüsseler EU-Gipfel Ende Juni 2012 Italien und Spanien unter Mitwirkung Frankreichs verdeckt zusammen, um so die deutsche Kanzlerin in die Schranken zu weisen und unserem Land zu zeigen, man wolle auch künftig nicht am deutschen Wesen genesen.

Mit all dem vor Augen ist kaum an der Einsicht vorbeizukommen, dass für die absehbare Zukunft der Intergouvernementalismus das wichtigste Politikmuster in der EU sein wird - zwar nicht „im Normalbetrieb“, sehr wohl aber in turbulenten Zeiten. Kommt es aber nicht zum Durchbruch hin zum vollendeten Supranationalismus mit verlässlicher Kontrolle europäischer Politik durch ein bevölkerungsproportionales Europäisches Parlament, so darf gerade der Bundestag unserer Regierung nicht mehr so gutwillig wie in der Vergangenheit freie Hand lassen. Erst recht darf er sich nicht durch europäisch oder gouvernemental erzeugten Zeitdruck um die ihm zustehenden Mitgestaltungsmöglichkeiten bringen lassen. ${ }^{9}$ Gerade darum geht es bei etlichen Verfahren, die Bundestagsabgeordne-

9 Falls auch das Parlament sich ,,von den Märkten getrieben“ fühlt, hat es zu hinterfragen, ob denn noch das grundsätzlich richtige Verhältnis zwischen Politik und Märkten besteht, und hat gegebenenfalls die nötigen Korrekturen an diesem Verhältnis herbeizuführen. Die bei der Euro-Rettungspolitik ganz offenkundig verletzte Grundregel lässt sich dahingehend formulieren, dass Politik ,,von außen“ und über längere Zeiten die Rahmenbedingungen von Märkten ordnungspolitisch zu setzen hat, das Marktgeschehen aber nicht kurzfristig durch eigene Marktteilnahme zur prozesspolitischen Herbeiführung erwünschter Marktergebnisse manipulieren darf. Im letzteren Fall werden gewinnorientierte Marktteilnehmer regel- 
te bzw. eine Bundestagsfraktion vor dem Bundesverfassungsgericht gegen die Bundesregierung angestrengt haben. Dabei zeigte sich: „Es gibt noch Richter in Karlsruhe!“10 - und zwar solche, die sowohl den Bundestag als auch den deutschen Staat für den Kampf gegen Einbußen an Macht und Demokratie stärken. $\mathrm{Ob}$ sich die Rolle des Bundesverfassungsgerichts als „Hüter demokratischer Staatlichkeit“" verstetigen wird, müssen die noch kommenden Urteile zeigen, zumal jene zu den fünf Verfassungsklagen gegen den Euro-Rettungsschirm und den europäischen Fiskalpakt. Doch vorsichtshalber sollte der Bundestag seine Verantwortung für die deutsche Eigenstaatlichkeit und Demokratie nicht Mal um Mal ans Bundesverfassungsgericht delegieren, sondern sie selbst wahrnehmen. Was wäre zu diesem Zweck zu tun?

Erstens sollte der Bundestag verhindern, dass es die von seinen Mehrheitsentscheidungen abhängige Bundesregierung letztlich vertragswidrig, doch faktisch rechtswirksam, zu einer - wie immer ausgestalteten - Vergemeinschaftung der Staatsschulden in der Eurozone kommen lässt. Dies zöge nämlich zunächst die finanzielle Überforderung Deutschlands, sodann das Verdorren der europäischen Gesinnung der allermeisten Deutschen nach sich, am Ende sogar - der fahrlässig herbeigeführten Not gehorchend - mancherlei Vertragsbrüche unseres Landes. Das europäische Einigungswerk läge dann in Ruinen, und Deutschland gälte wie zu kaiserlichen Zeiten als Europas unzuverlässiger Störenfried.

Zweitens muss der Bundestag, hierin ohnehin schon vom Verfassungsgericht bestärkt, auch in der Staatspraxis durchsetzen, dass es nie zur Auszahlung deutscher Haushaltsmittel im Rahmen der Euro-Rettungspolitik kommt, ohne dass dem ein konstitutiver Beschluss des Bundestages vorausgegangen ist. Einerseits wird dadurch gesichert, dass das Recht der Haushaltsfestsetzung - und somit der Kern von Staatlichkeit in Friedenszeiten - beim Parlament verbleibt. Andererseits wird nur so gewährleistet, dass die Bevölkerung erfährt, aus welchen Gründen und zu welchen Zwecken wieviel Geld, das man innerstaatlich gut brauchen könnte, an welchen anderen Staat oder welche andere Institution geht. Nur redliche Kommunikation darüber aber sichert europäischer Solidarität innerdeutsche Legitimation.

recht zu Trittbrettfahrertaktiken provoziert, was über Anschlussreaktionen der Politik das ganze Geschehen nur noch weiter beschleunigt und immer weniger kontrollierbar sowie beherrschbar macht.

10 So die Abwandlung der sprichwörtlichen Bemerkung eines legendären Brandenburger Müllers anlässlich eines Streits mit Friedrich dem Großen um die Mühle beim Schloss Sanssouci. 
Drittens sollte der Bundestag über die aktuellen Herausforderungen hinausblicken. Vor allem sollte er sich an die Schaffung jener plebiszitären Instrumente machen, welcher Deutschland beim Übergang zu mehr europäischer Supranationalität ohnehin bedürfen wird und die bereits jetzt beim Versuch helfen könnten, dem deutschen Volk solche Lasten zu ersparen, die es einfach nicht tragen will. Letzteres ,,aus übergeordneten Gesichtspunkten“ zu unterlassen, läuft auf nichts anderes hinaus als auf ,wohlmeinenden Autoritarismus“. Ein solcher aber passt nicht zu einer Gemeinschaft demokratischer Staaten. Hinsichtlich der Ausgestaltung des passenden plebiszitären Werkzeugs wäre es für Deutschlands repräsentative Demokratie wohl am besten, das - nicht nur für europapolitische Entscheidungen einsetzbare - Instrument eines fakultativen Referendums über alle Bundesgesetze einzuführen.

Es funktioniert so: Innerhalb einer ausreichend bemessenen Frist kann eine Referendumsinitiative - üblicherweise getragen von Parteien, Verbänden oder sonstigen zivilgesellschaftlichen Vereinigungen, in der Regel unterstützt oder bekämpft von konkurrierenden Massenmedien - sich an den meist vorab schon angekündigten Versuch machen, eine gesetzlich festgelegte und auf größeres Bevölkerungsinteresse hinweisende Anzahl von Unterschriften zu sammeln. Ist das gelungen, so findet eine Volksabstimmung statt, durch die das vom Parlament beschlossene, doch vom Bundespräsidenten noch nicht ausgefertigte Gesetz aufgehoben werden kann. Allein schon die Verfügbarkeit dieses Instruments, und gar erst seine wirkungsvolle Anwendbarkeit im Fall versuchten „Regierens gegen die Bürger“, änderte zweifellos viele politische Willensbildungsprozesse. Damit es möglichst nicht zum Missbrauch dieses Instruments durch die politische Klasse kommt, muss freilich ausgeschlossen werden, dass ein solches fakultatives Referendum von einem Verfassungsorgan herbeigeführt werden kann. ${ }^{11}$

Ein solches Verfahren ist gewiss besser, als dem Volk mit einer womöglich manipulativen Entscheidungsfrage folgender Art $\mathrm{zu}$ kommen: „Wollen Sie, dass ...?“. Auch hätte weiterhin, ganz wie es dem bewährten Repräsentationsprinzip entspricht, die Regierung und das sie unterstützende Parlament in jedem Fall das „erste Wort“. Das entscheidende letzte Wort aber, wann immer von einem nennenswerten Teil der Wählerschaft gewünscht, läge beim Volk. Gerade so muss es in einer Demokratie auch sein. Und wenn das Volk „Obstruktion“

11 S. Patzelt, W.J.: Welche plebiszitären Instrumente könnten wir brauchen? Einige systematische Überlegungen, in: Jahrbuch für direkte Demokratie 2010, Baden-Baden, 2011, 63-106. 
betreibt oder ,rasche Entscheidungen“ behindert? Dann klären zwei Gegenfragen die Lage. Darf in einer Demokratie das Volk wohl nur das wollen, was ihm seine Führer empfehlen? Und darf sich wohl nur ein Parlament bei der Gesetzgebung die nötige Zeit nehmen? Gewiss sind bei der Einführung plebiszitärer Instrumente schädliche Nebenwirkungen möglichst auszuschließen. Doch guten Willen vorausgesetzt, lässt sich das Instrument des fakultativen Gesetzesreferendums staatspolitisch klug und europapolitisch sinnvoll ausgestalten.

Viertens sollte der Bundestag sich mit langem Atem daran machen, durch Entschließungen und Kopplungsgeschäfte darauf hinzuwirken, dass eines Tages die - auch vom Bundesverfassungsgericht mehrfach benannte - wichtigste Voraussetzung für mehr Supranationalität in Europa und für mehr Macht des Europäischen Parlaments geschaffen wird: nämlich eine Zusammensetzung dieser Volks- oder Völkervertretung dahingehend, dass die Stimmen aller Wähler ungeachtet ihrer Staatsangehörigkeit bei Europawahlen das gleiche Erfolgsgewicht besitzen.

Fünftens sollte der Bundestag unbedingt verhindern, dass es zu falschen Weichenstellungen bei der immer wieder nötigen Weiterentwicklung des supranationalen Regierungssystems der EU kommt. Vor allem wäre zu unterbinden, dass die Direktwahl eines Europäischen Präsidenten eingeführt wird. Mit ihr entstünde nämlich entweder eine recht machtlose, dann aber auch überflüssige und deshalb Zweifel an ernstgemeinter Demokratie nährende Institution. Oder es wäre, bei entsprechend großer Kompetenzausstattung des Präsidentenamtes, der Weg hin zu einem präsidentiellen Regierungssystem auf EU-Ebene vorgebahnt. Das aber verspricht auf EU-Ebene ebenso wenig zu funktionieren wie eine Mischung aus präsidentiellem und parlamentarischem Regierungssystem. Darauf mag man zwar in einer politischen Ordnung ,sui generis“ wie der europäischen manche Hoffnung setzen. Doch in der EU ist schon zu Normalzeiten das Regieren so schwierig, dass man nicht so tollkühn sein sollte, dem auch noch die Reibungsverluste eines problematischen Regierungssystems anzufügen. Hingegen sollte der Bundestag, wann immer opportun, die Bundesregierung zu Positionen drängen, die auf eine Parlamentarisierung der Europäischen Kommission hinauslaufen, also auf die Einführung des parlamentarischen Regierungssystems in der EU.

Aus einer tragfähigen Antwort auf die Frage nach der richtigen Ausübung von Deutschlands ,europäischem Beruf“, vom Parlament endlich bis auf den Grund gehend zu erörtern, folgt für den Deutschen Bundestag also höchst Konkretes. Was immer davon das Parlament ernsthaft anpacken will: Wichtig ist, alle Ein- 
zelmaßnahmen von jenem größeren Ganzen her zu bedenken, dem sie nachhaltig dienen sollen. Dieses Ganze ist eine stabile europäische Friedensordnung, gesteuert - in subsidiärer Arbeitsteilung mit den Nationalstaaten - von einem demokratisch effektiv kontrollierten europäischen Regierungssystem, die Deutschland für alle absehbare Zeit von seinem geopolitischen Dilemma befreit. Das Mittel zu diesem Zweck ist für die kommenden Jahre aber nicht „,mehr Supranationalität“, sondern „,besser kontrollierter Intergouvernementalismus“. 